\title{
SPECIAL POINTS ON FIRST ORDER PARTIAL DIFFERENTIAL EQUATIONS AND THE DEFORMATIONS OF SOLUTIONS
}

\author{
MAREK KOSSOWSKI
}

\begin{abstract}
The object of this paper is to identify four cases of special behavior in a class of first order PDE for a real valued function. (The class of PDE may be thought of as perturbation of PDE with singular solutions.) In each case we show how invariants of the PDE determine properties of solutions. The properties of solutions examined here are the structure of critical points and singularities induced by cotangent projection. These properties are described in the sense of constructing local models for solutions and characterizing their behavior under small deformations. We will find two cases where deformations exhibit bifurcation phenomena, and describe generic deformations.
\end{abstract}

The objective of this paper is to identify four cases of special behavior in first order PDE for a real valued function. In each case we show how invariants of the PDE determine properties of solutions. The properties of solutions examined here are the structure of critical points and singularities induced by cotangent projection. These properties are described in the sense of constructing local models for solutions and characterizing their behavior under small deformations. We will find two cases where deformations exhibit bifurcation phenomena, and describe generic deformations. By taking a geometric viewpoint these cases can be presented in a unified manner.

The geometric theory of partial differential equations views a PDE as a locus of points in an appropriate jet bundle (usually this locus is assumed to be the image of a smooth map $E \hookrightarrow J)$. Here we consider the simplest case of a single first order PDE for a real valued function on a smooth manifold $M$ (i.e. $E \hookrightarrow J^{1}(M, \mathbf{R})$ is a hypersurface). The interaction of this hypersurface with natural structures on $J^{1}(M, \mathbf{R})$ determines properties of the PDE and its solutions. We will find that there is a natural smooth variety lying in the hypersurface $E$. Each of the cases of special behavior corresponds to points in this variety and are identified via geometric conditions on the hypersurface $E$. There is a hierarchy among these points in the sense that a generic deformation of a solution containing a point of Case 4 will contain points of Cases 1 and 3 . The first two cases of points are the natural place to find critical points of solutions. Points of Cases 3 and 4 may be thought of as "perturbed singular solutions." (For a discussion of first order PDE with singular solutions we refer the reader to $[\mathbf{K}]$.)

We first identify the general class of PDE with which we will work, prove an existence theorem for deformations of solutions and identify the variety in which

Received by the editors November 12, 1984 and, in revised form, June 3, 1986.

1980 Mathematics Subject Classification (1985 Revision). Primary 35A30, 35B32, 35F20; Secondary 35C99.

Research partially supported by NSF Grant DMS 83-52009. 
the special points lie. Upon identifying a special point, we then produce a local model for a solution which contains such a point and satisfies generic conditions. (These conditions are given in terms of invariants extracted from the PDE.) After describing the behavior of a small deformation of such a solution we repeat this procedure with the next case. The presentation will be axiomatic. The reader may find it helpful to consult the examples at the end of the paper.

The approach implicit in this work is the combination of the elementary theory of differential systems (i.e. the Pfaff Theorem: local models for 1-forms) with the ideas of $C^{\infty}$ singularity theory. We intend to consider solutions as functions. Thus we use the equivalence relation induced by diffeomorphisms of $M$. It is here that this work differs from much of the geometrical literature on first order PDE. Classically weaker notions of equivalence were used (see $[\mathbf{L}, \mathbf{A}]$ ). It is of note that with respect to this stronger equivalence the PDE considered here differ significantly from classical Hamilton Jacobi type.

The author would like to thank R. B. Gardner, J. Damon, M. Schlessinger, J. Stasheff, and G. Thompson for inspiration.

Preliminaries. The symbols $C^{\infty}(M, \mathbf{R}), \mathfrak{X}(M)$ will denote smooth functions and vector fields on $M$. A subscript $x \in M$ will denote the germ of such an object at $x . \mathcal{M}_{x}(M) \subset C_{x}^{\infty}(M, \mathbf{R})$ will denote the maximal ideal in the ring of germs of functions at $x \in M$. Given a smooth map $\phi: M \rightarrow N$ between manifolds we will use the notation $\phi:(M, x) \rightarrow(N, \phi(x))$ to denote its germ at $x \in M$. In that the bulk of our work will depend only upon the germs of the mappings involved we will not make constant reference to this fact. All objects are to be taken as smooth. If $X$ is a vector field on $M$ and $\omega$ a 1 -form on $M$ we will write $\langle X \mid \omega\rangle$ or $X(\omega)$ for their pairing. Also the iterated pairing $X(X(\omega))$ may be written $\left\langle X^{2} \mid \omega\right\rangle$. $\operatorname{Anhil}(\omega)$ will denote the distribution of hyperplanes which $\omega$ annihilates. The symbol $\pitchfork$ will denote transverse intersection of submanifolds. The symbol $\rfloor$ will denote contraction of a form by a vector field. We then have the H. Cartan formula, $\left.\left.\mathcal{L}_{X} \omega=d(\omega\lrcorner X\right)+(d \omega)\right\lrcorner X$, for the Lie derivative of $\omega$.

Given $M$ and $f: M \rightarrow \mathbf{R}$ we have the associated 1-jet section $j^{1} f: M \rightarrow$ $J^{1}(M, \mathbf{R})$. The contact distribution on $J^{1}(M, \mathbf{R})$ is a codimension one distribution. Its role is to characterize those $m$-dimensional submanifolds of $J^{1}(M, \mathbf{R})$ which are the image of a 1-jet section. $J^{1}(M, \mathbf{R})$ also possesses several fibrations. Namely,

$$
\begin{array}{rlrl}
\pi_{T^{*} M}: J^{1}(M, \mathbf{R}) & \rightarrow T^{*} M, & & j_{x}^{1} f \rightarrow d_{x} f, \\
\pi_{M \times \mathbf{R}}: J^{1}(M, \mathbf{R}) & \rightarrow M \times \mathbf{R}, & j_{x}^{1} f \rightarrow(x, f(x)), \\
\pi_{\mathbf{R}}: J^{1}(M, \mathbf{R}) \rightarrow \mathbf{R}, & j_{x}^{1} f \rightarrow f(x) .
\end{array}
$$

(We will write $\pi$ for the projection of $T^{*} M$ onto $M$.) Finally $J^{1}(M, \mathbf{R})$ contains the $(m+1)$-dimensional submanifold of constant 1-jets,

$$
C J=\left\{j_{x}^{1} f \mid x \in M, f \text { is constant }\right\} .
$$

We are interested in the interaction of $E$ with the above structures on $J^{1}(M, \mathbf{R})$. In particular if the tangent space to $E$ agrees with the contact distribution at $j_{x}^{1} f$ we say such a point is a contact singularity of $E$. If $\pi_{M \times \mathbf{R}}: E \rightarrow M \times \mathbf{R}$ fails to have a maximal rank we refer to such a point as a $\pi_{M \times \mathbf{R}}$-singularity. Finally the intersection of $E$ with $C J$ will typically be an $m$-dimensional manifold. 
Throughout this paper we will make the assumption that the map $\pi_{T^{*} M}$ gives a global diffeomorphism of $E$ with $T^{*} M$. Since $\pi_{\mathbf{R}}$ yields a trivialization of $J^{1}(M, \mathbf{R})$ over $T^{*} M$ we see that $E$ is then the graph of a function $h: T^{*} M \rightarrow \mathbf{R}$. Thus with this assumption we may present such PDE as $f(x)=h\left(d_{x} f\right)$ or as the image of

$$
G(h): T^{*} M \rightarrow J^{1}(M, \mathbf{R}), \quad d_{x} f \rightarrow\left(d_{x} f, h\left(d_{x} f\right)\right) .
$$

DEFINITION 1.1. A graphlike first order PDE on $M$ is a pair $(M, h)$ where $h \in C^{\infty}\left(T^{*} M, \mathbf{R}\right)$. We will say that $(M, h)$ is equivalent to $\left(M^{\prime}, h^{\prime}\right)$ if there exists a diffeomorphism $\phi: M \rightarrow M^{\prime}$ such that $\left(\phi^{*}\right)^{*} h^{\prime}=h$.

Given $(M, h)$ we now pull back the relevant structures of $J^{1}(M, \mathbf{R})$ to $T^{*} M$. Writing $\theta$ for the canonical 1-form on $T^{*} M$ we may write $\omega_{h}=d h-\theta\left(d=d^{T^{*} M}\right)$ and describe the pull back of the contact distribution as Anhil $\left(\omega_{h}\right)$. Now $\pi: T^{*} M \rightarrow$ $M$ and $h: T^{*} M \rightarrow \mathbf{R}$ are associated with fibrations of $J^{1}(M, \mathbf{R})$. Finally $E \cap C J$ corresponds to $Z$, the zero section of $T^{*} M$. (Also, $v_{x} \in T^{*} M$ corresponds to a point of contact singularity iff $\left.\omega_{h}\right|_{v_{x}}=0$.)

Given $(M, h)$ we define a solution to be a smooth immersion $s: L \rightarrow T^{*} M$ of an $m$-dimensional manifold $L$ such that $s^{*} \omega_{h}=0$. If $(\pi \circ s)_{*}$ has full rank at $l \in L$ then (via the inverse function theorem) $s$ determines a unique germ of a real-valued function at $\pi \circ s(l)$. If this condition fails to hold then $s$ determines a "multiple valued function." Notice that in either case $s^{*} h$ is well defined and contains the information of the solution as a function. That is to say, in either case we may define the graph of $s$ to be the map

$$
\operatorname{graph}(s): L \rightarrow M \times \mathbf{R}, \quad l \rightarrow\left(\pi \circ s(l), s^{*} h(l)\right) .
$$

Given $(M, h)$ let $X_{h}$ denote the characteristic vector field on $T^{*} M$ defined by the condition $\left.\omega_{h}=X_{h}\right\rfloor d \theta . H_{t}$ will denote its flow. It follows that $\mathcal{L}_{X_{h}} \omega_{h}=\omega_{h}$. Since solutions are Lagrangian submanifolds (i.e. maximally isotropic) of $T^{*} M$, we see that $X_{h}$ is tangent to every solution. Given a solution $s: L \rightarrow T^{*} M$ we write $s^{*} X_{h}$ for the vector field on $L$ which is $s$-related to $X_{h}$. Now we consider the smooth variety, $V[h]=\left\{v_{x} \in T^{*} M \mid\left\langle X_{h} \mid h\right\rangle_{v_{x}}=0\right\}$. Our objective is to understand the behavior of $\pi \circ s$ and $s^{*} h$ for solutions which contain certain special points in $V[h]$. (Specifically we are interested in local models for $s^{*} h, \pi \circ s$, and their behavior under deformation.) Here "special" refers to geometric properties of $V[h]$.

The following is an existence theorem for solutions and their deformation. Our proof is based on the Pfaff Theorem which we assume familiar to the reader; see Bryant, Chern, Griffiths, or R. B. Gardner.

Proposition 1.2. Given $(M, h), v_{x} \in T^{*} M$ with $\left.\omega_{h}\right|_{v_{x}} \neq 0$, let

$$
V \subset \operatorname{Anhil}\left(\omega_{h}\right) \subset T_{v_{x}} T^{*} M
$$

be a Lagrangian subspace which contains $\left.X_{h}\right|_{v_{x}}$. If $c:(\mathbf{R}, 0) \rightarrow\left(T^{*} M, v_{x}\right)$ is a smooth curve with $\omega_{h}(\dot{c}(0)) \neq 0$ then there exists (a germ of) a 1-parameter family of solutions $s_{u}:\left(\mathbf{R}^{m} \times \mathbf{R}, 0\right) \rightarrow\left(T^{*} M, v_{x}\right)$ such that $\left(s_{0}\right)_{*} T_{0} \mathbf{R}^{m}=V$ and $s_{u}(0)=c(u)$.

PROOF. Let $\psi:\left(\mathbf{R}^{2 m-1}, 0\right) \rightarrow\left(T^{*} M, v_{x}\right)$ be transverse to $X_{h}$ at $v_{x}$ and contain the image of $c$. We may write $V=X_{h} \oplus \psi_{*} W$ with $W$ a unique $m-1$ dimensional subspace of $T_{0} \mathbf{R}^{2 m-1}$. Now the Pfaff Theorem enables us to artificially impose a $J^{1}\left(\mathbf{R}^{m-1}, \mathbf{R}\right)$ structure on a neighborhood of 0 in $\mathbf{R}^{2 m-1}$ so that $\operatorname{Anhil}\left(\psi^{*} \omega_{h}\right)$ agrees with the contact distribution. Since every symplectomorphism of $T^{*} \mathbf{R}^{m-1}$ 
has a lift to a contact-morphism of $J^{1}\left(\mathbf{R}^{m-1}, \mathbf{R}\right)$, symplectic geometry allows us to assume that $W$ is the tangent space to the image of $j^{1}(0)$ at 0 . Here $0: \mathbf{R}^{m-1} \rightarrow \mathbf{R}$ is the constant function. Now let $g_{u}$ be a 1-parameter family of $\mathbf{R}$ valued functions on $\mathbf{R}^{m-1}$ such that $\left(j_{0}^{1} g_{0}\right)_{*} T_{0} \mathbf{R}^{m-1}=W$ and $\psi \circ g_{u}(0)=c(u)$. Then $s_{u}$ is given by $H_{t} \circ \psi \circ j^{1} g_{u}$.

2. Solutions containing special points of $V[h]$. We now turn to the central results of this paper. We assume throughout the remainder of the paper that $(M, h)$ has no contact singularities on $Z$. This is equivalent to the condition $d h \neq 0$ on $Z$. (We will abuse notation and let $Z$ denote the zero section both as a map and as a set.) A computation shows that $\left\langle X_{h} \mid h\right\rangle=\left\langle V^{c} \mid h\right\rangle$ where $V^{c}$ is the canonical vector field on $T^{*} M$ associated with the natural $\mathbf{R}$-action. It follows that $Z \subset$ $V[h]$. Thus we begin our discussion with solutions which intersect the zero section. Observe that if $s:\left(\mathbf{R}^{m}, 0\right) \rightarrow\left(T^{*} M, o_{x}\right)$ is such a germ then $s^{*} \omega_{h}=0$ implies that $d\left(s^{*} h\right)=\left(s^{*} \theta\right)=0$ at 0 . Further $\operatorname{Hess}_{o}\left(s^{*} h\right)=s^{*}\left(\odot \operatorname{Hess}_{o_{x}} \theta\right)$, where $\odot \operatorname{Hess}_{o_{x}} \theta$ is the symmetric part of the Hessian of $\theta$. (Recall that $\operatorname{Hess}_{o_{x}} \theta(X, Y)=X(\theta(Y))_{o_{x}}$ is a nonsymmetric $(0,2)$ tensor on $T_{o_{x}} T^{*} M$. Its skew part agrees with $d \theta$ over $Z$.)

We extract the relevant information from $(M, h)$ as follows. Given $x \in M$ restrict $h$ to $T_{x}^{*} M$ and construct its affine approximation at the origin. Varying $x$ we may view this as a function ${ }_{A} h \in C^{\infty}\left(T^{*} M, \mathbf{R}\right)$. Using the isomorphism of $T_{p} M$ and $\left(T_{p}^{*} M\right)^{*}$ we may identify ${ }_{A} h$ with a pair, ${ }_{A} k \in C^{\infty}(M, \mathbf{R})$ and ${ }_{A} Y \in \mathfrak{X}(M)$, where ${ }_{A} h\left(d_{x} f\right)={ }_{A} k(x)+\left\langle{ }_{A} Y \mid d f\right\rangle_{x}$. We make the following identifications.

Proposition 2.1. Given $(M, h)$ :

(a) $\left.X_{h}\right|_{o_{x}}=Z_{*}\left({ }_{A} Y\right)_{x}-d\left({ }_{A} k\right)_{x}$. Here $Z$ denotes the zero section as a map $Z: M \rightarrow T^{*} M$ and $d_{A} k$ is viewed (via the identification of a vector space with its tangent spaces) as a vector tangent to the fiber of $T^{*} M$ at $o_{x}$.

(b) $\odot \operatorname{Hess}_{o_{x}} \theta\left(X_{h}, X_{h}\right)=-\left\langle\left.{ }_{A} Y\right|_{A} k\right\rangle_{x}$.

(c) $\left\langle X_{h}^{3} \mid h\right\rangle_{o_{x}}=\left\langle\left.{ }_{A} Y^{2}\right|_{A} k\right\rangle_{x}$.

Proof. Choosing local coordinates $\left(x_{i}\right)$ for $M$ and adapted coordinates $\left(x_{i}, p_{i}, z\right)$ for $J^{1}(M, \mathbf{R})$ we may write the following local representatives.

$$
\begin{aligned}
& X_{h}=h_{p_{i}} \frac{\partial}{\partial x_{i}}+\left(p_{i}-h_{x_{i}}\right) \frac{\partial}{\partial p_{i}} \quad\left(h_{p_{i}}=\frac{\partial h}{\partial p_{i}}\right), \\
& { }_{A} Y(x)=h_{p_{i}}(x, 0) \frac{\partial}{\partial x_{i}}, \quad d_{A} k(x)=h_{x_{i}}(x, 0) d x_{i},
\end{aligned}
$$

$\odot \operatorname{Hess}_{o_{x}} \theta=d x_{i} \odot d p_{i} \quad$ (symmetric tensor product).

(a), (b), and (c) now follow directly.

CASE 1. Let $o_{x} \in T^{*} M$ be such that $d_{A} k(x) \neq 0$ and ${ }_{A} Y(x) \neq 0$. It follows from Proposition 2.1(a) that $\left.X_{h}\right|_{o_{x}}$ is not tangent to either the fiber of $T^{*} M$ or the zero section.

THEOREM 2.2. Let $s:\left(\mathbf{R}^{m}, 0\right) \rightarrow\left(T^{*} M, o_{x}\right)$ be the germ of a solution with $o_{x}$ of Case 1.

(a) The following is an open dense condition on $j_{0}^{1} s$ :

(i) $s \pitchfork Z$ ( $\pitchfork$ denotes nonempty transverse intersection),

(ii) $s \pitchfork$ fiber of $T^{*} M$. 
(b) For $s$ as in (a),

(i) if $\left\langle\left.{ }_{A} Y\right|_{A} k\right\rangle_{x}>0$ then Hess $s^{*} h$ has type $(r, s)$ with $s>0$,

(ii) if $\left\langle\left._{A} Y\right|_{A} k\right\rangle_{x}<0$ then Hess $s^{*} h$ has type $(r, s)$ with $r>0$,

(iii) if $\left\langle{ }_{A} Y \mid{ }_{A} k\right\rangle=0$ then Hess $s^{*} h$ has type $(r, s)$ with $r-s \neq \pm m$.

(c) Let $s_{u}$ be a deformation such that $s_{0}$ is as in (a); then the deformation is trivial (i.e. $s_{u}=\left(\phi^{*}\right)^{*} s_{0}$ for some $\phi \in \operatorname{Diff}_{x}(M)$ and all $u$ sufficiently small).

PROOF. (a) Left to the reader. Compare the proof of Case 3(a) for an illustration of such an argument.

(b) Since $s \pitchfork Z$, Hess $_{o} s^{*} h$ has maximal rank. Observing that

$$
\operatorname{Hess}_{o} s^{*} h\left(s^{*} X_{h}, s^{*} X_{h}\right)=\odot \operatorname{Hess}_{o_{x}} \theta\left(X_{h}, X_{h}\right)
$$

we are finished.

(c) By elementary transversality theory $s_{u}$ is transverse to $Z$ and to the fiber for sufficiently small $u$. Thus $s_{u}$ may be parametrized as a section $d f_{u}$ of $T^{*} M$. Since $f$ is morse $f_{u}$ will also be morse of the same signature.

CASE 2. Let $o_{x} \in T^{*} M$ be such that $d_{A} k(x)=0, \operatorname{Hess}_{x}\left({ }_{A} k\right)$ is nonsingular, ${ }_{A} Y(x) \neq 0$, and $\left\langle_{A} Y^{2} \mid{ }_{A} k\right\rangle \neq 0$. It follows that $X_{h}$ is tangent to the zero section at $o_{x}$. A computation shows that $\operatorname{Anhil}\left(\omega_{h}\right)$ and $X_{h}^{\perp}(\perp$ relative to $\odot$ Hess $\theta)$ agree as subspaces of $T_{o_{x}} T^{*} M$. Thus $s^{*} X_{h}$ lies in the radical (i.e. degenerate subspace) of Hess $_{o} s^{*} h$ for any solution germ $s:\left(\mathbf{R}^{m}, 0\right) \rightarrow\left(T^{*} M, o_{x}\right)$.

THEOREM 2.3. Let $s:\left(\mathbf{R}^{m}, 0\right) \rightarrow\left(T^{*} M, o_{x}\right)$ be the germ of a solution with $o_{x}$ of Case 2 .

(a) The following is an open dense condition on $j_{0}^{1} s$ :

(i) $s \pitchfork$ fiber,

(ii) Hess $s^{*} h$ has rank $m-1$.

(b) For $s$ as in (a),

(i) the singularity of $s^{*} h$ is isolated,

(ii) the solution admits local model df where $f=x_{1}^{3}+\sum_{2}^{m} \pm x_{i}^{2}$.

(c) Let $s_{u}$ be a deformation such that $s_{u}$ is as in (a) and $\left(s_{u}\right)_{*}(\partial / \partial u)$ is transverse to variety $V[h]\left(o_{x}\right.$ is a regular point of $\left.V[h]\right)$; then

(i) for $u>0 s_{u}^{*} h$ has no critical points,

(ii) for $u<0 s_{u}^{*} h$ has two morse critical points with types $(r+1, s)$ and $\left.r, s+1\right)$.

PROOF. (a) Left to the reader; compare the proof of Case 3(a). Now consider

(b) and (c) Since $s$ is transverse to the fiber it may be parametrized as a section $s=d f$; hence, $s^{*} h=f$. Our proofs of (b) and (c) will be based on the following observation. We claim that there exist coordinates so that $s_{u}^{*} h-s_{u}^{*} h(0)$ is represented by

$$
x_{1}\left[u a(x, u)+\sum_{1}^{m} x_{i} x_{j} b_{i j}(x, u)\right]+\sum_{i=2}^{m} \pm x_{i}^{2},
$$

with $a(0,0) \neq 0$ and $b_{11}(0,0) \neq 0$. (Note that $\left(s_{u}^{*} h-s_{u}^{*} h(0)\right)$ evaluated at $x \in \mathbf{R}^{m}$ is $s_{u}^{*} h(x)-s_{u}^{*} h(0)$.)

Observe that the condition $\left(s_{u}\right)_{*}(\partial / \partial u) \pitchfork V[h]$ implies that $s_{u}^{*} h-s_{u}^{*} h(0)$ has a morse singularity at the origin of $\mathbf{R}^{m+1}$. Thus we may choose coordinates such that $s_{u}^{*} h-s_{u}^{*} h(0)$ is represented by $z_{1} v+\sum_{i=2}^{m} z_{i}^{2}$ with the tangent space to the 
leaf $u=0$ spanned by $\partial / \partial z_{1} \cdots \partial / \partial z_{m}$ at the origin. Thus the foliation $u=$ const can be parametrized by $\phi:\left(\mathbf{R}^{m} \times \mathbf{R}, 0\right) \rightarrow\left(\mathbf{R}^{m} \times \mathbf{R}, 0\right)$ :

$$
\phi^{*}\left(z_{i}\right)=x_{i}, \quad i=1, \ldots, m, \quad \phi^{*}(v)=b_{u}(x),
$$

where $b_{u}(x)=u a(x, u)+\sum_{i=1}^{m} x_{i} x_{j} b_{i j}(x, u)$. Clearly $a(0,0) \neq 0$. Since $s^{*} X_{h}$ is represented by $\partial / \partial x_{1}$ at 0 , and $\left\langle X_{h}^{3} \mid h\right\rangle_{o_{x}} \neq 0$ we have $b_{11}(0,0) \neq 0$.

To prove (b) set $s=s_{0}$ in the above and observe

$$
\frac{\partial}{\partial x_{i}} s^{*} h \equiv \pm 2 x_{i} \bmod \mathcal{M}_{0}^{2}\left(\mathbf{R}^{m}\right)
$$

for $i=2, \ldots, m$. Thus the variety $\left\{\left(\partial / \partial x_{i}\right) s^{*} h=0 \mid i=2, \ldots, m\right\}$ is an immersed curve tangent to $\partial / \partial x_{1}$ at 0 . Now since $b_{11}(0) \neq 0$ the function $\left(\partial / \partial x_{1}\right) s^{*} h$ restricted to this curve is morse at 0 . The singularity is isolated. Now we may apply the relative morse lemma and the theorem of Mather-Tougeron (see Guillemin, Sternberg, p. 16) to construct the local model.

To prove (c) we return to the representative of $s_{u}^{*} h$ constructed above and isolate the curve of critical points. Observe that

$$
\frac{\partial}{\partial x_{1}} s_{u}^{*} h \equiv u a \bmod \mathcal{M}_{0}^{2}\left(\mathbf{R}^{m+1}\right) \quad \text { and } \quad \frac{\partial}{\partial x_{i}} s_{u}^{*} h \equiv \pm 2 x_{i} \bmod \mathcal{M}_{0}^{2}\left(\mathbf{R}^{m+1}\right)
$$

for $i=2, \ldots, m$. Thus the variety $\left\{\left(\partial / \partial x_{i}\right) s_{u}^{*} h=0 \mid i=1, \ldots, m\right\}$ is an immersed curve which is tangent to the leaf $u=0$ in the direction $\partial / \partial x_{1}$ at 0 . We may construct an immersion $\tau:\left(\mathbf{R}^{2}, 0\right) \rightarrow\left(\mathbf{R}^{m} \times \mathbf{R}, 0\right)$,

$$
\tau^{*}(u)=u, \quad \tau^{*}\left(x_{i}\right)=x_{i}(u, v), \quad i=1, \ldots, m,
$$

with the image of $\tau$ containing this curve and $\tau_{*}(\partial / \partial v)=\partial / \partial x_{1}$ at 0 . (This can be done by suspending the curve in a vector field, flowing off the $u$-axis, and then reparametrizing so that the foliation is again given by $u=c$. Now $\tau^{*}\left(s_{u}^{*} h-s_{u}^{*} h(0)\right)$ is represented by

$$
u v \bar{a}(v, u)+v^{3} \bar{b}(v, u)
$$

with $\bar{a}(0,0)$ and $\bar{b}(0,0) \neq 0$. The above curve of critical points corresponds to

$$
\frac{\partial}{\partial v}\left(\tau^{*}\left(s_{u} h-s_{u}^{*} h(0)\right)\right)=0
$$

and may be parametrized as $u=u(v)$ with $0=u(0)=\dot{u}(0)$. A calculation implies $\ddot{u}(0) \neq 0$. Thus the critical point bifurcates.

To understand the behavior of the signature along the curve of critical points we need only isolate the behavior of

$$
\frac{\partial^{2}}{\partial v \partial v}\left(\tau^{*}\left(s_{u}^{*} h-s_{u} h(0)\right)\right)
$$

But we have that

$$
\left.\frac{\partial^{3}}{\partial v \partial v \partial v}\left(\tau^{*}\left(s_{u}^{*} h-s_{u} h(0)\right)\right)\right|_{0,0}=\bar{b}(0,0) \neq 0,
$$

and we are finished.

CASE 3. Let $o_{x} \in T^{*} M$ be such that $d_{A} k(x) \neq 0,{ }_{A} Y(x)=0$. It follows that $X_{h}$ is tangent to the fiber of $T^{*} M$ at $o_{x}$. Thus no solution passing through $o_{x}$ can be presented as a section of $T^{*} M$. A computation shows that the map

$$
\pi_{M \times \mathbf{R}} \circ G(h): T^{*} M \rightarrow M \times \mathbf{R}, \quad d_{x} f \rightarrow\left(x, h\left(d_{x} f\right)\right),
$$


has rank $m$ at $o_{x}$. That is to say $o_{x}$ is a point of $\pi_{M \times \mathbf{R}}$-singularity. We deal with this by imposing generic assumptions on the structure of this singularity. We recall the notion of submersion with fold (see Golubitsky and Guillemin).

Let $g: Y \rightarrow X$ be a smooth map of manifolds with $\operatorname{Dim} Y=\operatorname{Dim} X+r, r \geq 0$, and let ${ }_{1} S \subset J^{1}(X, Y)$ denote the codimension $r+1$ submanifold of jets with rank one less than maximal.

DEFINITION 2.4. $g$ has a fold at $y \in Y$ if

(a) $j^{1} g: Y \rightarrow J^{1}(Y, X)$ is transverse to ${ }_{1} S$ at $y$,

(b) the fold locus $\left(j^{1} g\right)^{-1}\left({ }_{1} S\right)$ (a codimension $r+1$ submanifold of $Y$ ) has the property that its tangent space and $\left.\operatorname{ker} g_{*}\right|_{y}$ split $T_{y} Y$ (i.e., $T_{y}\left(j^{1} g\right)^{-1}\left({ }_{1} S\right)+$ $\left.\left.\operatorname{ker} g_{*}\right|_{y}=T_{y} Y\right)$.

If this condition (with $r=m-1$ ) is satisfied by $\pi_{M \times \mathbf{R}} \circ G(h)$ at a point of $\pi_{M \times \mathbf{R}^{-}}$ singularity we say $(M, h)$ is folded simply at this point. The kernel of $\left(\pi_{M \times \mathbf{R}} \circ G(h)\right)_{*}$ must then be tangent to the fiber. Hence the fold locus may be locally parametrized as the image of a 1 -form on $M$. (We will abuse notation and write $\tau_{F}$ for both the 1 -form on $M$, and its image as a map into $T^{*} M$.)

Here we will assume that the fold locus of $\pi_{M \times \mathbf{R}} \circ G(h)$ is given by a single 1 -form $\tau_{F}$ on $M$ and that there are no contact singularities on its image. This latter assumption is equivalent to the nonvanishing of $\omega_{h}$ when pulled back over $\tau_{F}: M \rightarrow T^{*} M$ (i.e. $\left.\tau_{F}^{*}\left(\omega_{h}\right) \neq 0\right)$.

On the other hand if the above condition (with $r=0$ ) is satisfied by $\pi \circ s$ at $l \in L$ we say the solution $s$ is folded simply at $l$. We will write $\mathrm{FL}(s)$ for $j^{1}(\pi \circ s)^{-1}\left({ }_{1} S\right)$, the fold locus of the solution.

Noting that $o_{x}$ is of Case 3 if $\tau_{F}(x)=0$ we will discuss solutions which intersect $\tau_{F}$ as a preliminary. (Recall that since $\left\langle X_{h} \mid h\right\rangle=\left\langle V^{c} \mid h\right\rangle$ it follows that $\tau_{F}$ is contained in the variety $V[h]$.) We extract the relevant information from $(M, h)$ as follows. Given $x \in M$ restrict $h$ to $T_{x}^{*} M$ and construct its quadratic approximation at the point $\tau_{F}(x)$. Varying $x$ we may view this as a function $Q_{Q} h \in C^{\infty}\left(T^{*} M, \mathbf{R}\right)$. We may identify ${ }_{Q} h$ with a triple, ${ }_{Q} k \in C^{\infty}(M, \mathbf{R}),{ }_{Q} Y \in \mathfrak{X}(M)$, and ${ }_{Q}\langle\rangle$ a section of $T M \odot T M$, where ${ }_{Q} h\left(d_{x} f\right)={ }_{Q} k(x)+\langle Y \mid d f\rangle_{x}+{ }_{Q}\langle d f, d f\rangle_{x}$. Since $(M, h)$ is folded simply the rank of $Q\langle\rangle$ is maximal. We will write ${ }^{Q}\langle\rangle$ for the associated pseudo-Riemannian metric. We make the following identifications.

PROPOSITION 2.5. (a) $\left.X_{h}\right|_{\tau_{F}}=\tau_{F}^{*}\left(\omega_{h}\right)$, where $\tau_{F}^{*}\left(\omega_{h}\right)$ is viewed as a vector tangent to the fiber of $T^{*} M$ at $\tau_{F}(x)$.

(b) $\left\langle X_{h}^{2} \mid h\right\rangle_{\tau_{F}(x)}={ }_{Q}\left\langle\tau_{F}, \tau_{F}^{*}\left(\omega_{h}\right)\right\rangle_{x}$.

(c) If $\tau_{F}(x)=0$ then $\left\langle X_{h}^{3} \mid h\right\rangle_{o_{x}}={ }_{Q}\left\langle d_{Q} k, d_{Q} k\right\rangle_{x}$.

PROOF. Using local representatives,

$$
\begin{aligned}
& \omega_{h}=\left(h_{x_{i}}-p_{i}\right) d x_{i}+h_{p_{i}} d p_{i}, \\
& \tau_{F}=a_{i}(x) d x_{i}, \quad \text { where } h_{p_{i}}\left(x_{j}, a_{j}(x)\right)=0, \\
& X_{h}=h_{p_{i}} \frac{\partial}{\partial x_{i}}+\left(p_{i}-h_{x_{i}}\right) \frac{\partial}{\partial p_{i}}, \\
& \left\langle X_{h} \mid h\right\rangle=p_{i} h_{p_{i}},
\end{aligned}
$$

(a), (b), and (c) follow directly.

The following is a basic property of solutions which intersect $\tau_{F}$. 
THEOREM 2.6. Let $(M, h)$ satisfy $\tau_{F}^{*} \omega_{h}(x) \neq 0$ and let

$$
s:\left(\mathbf{R}^{m}, 0\right) \rightarrow\left(T^{*} M, \tau_{F}(x)\right)
$$

be the germ of a solution. Then

(a) $\pi \circ s:\left(\mathbf{R}^{m}, 0\right) \rightarrow(M, x)$ has 1 -jet transverse to ${ }_{1} S$ if $(\pi \circ s)_{*}$ has rank $m-1$ at 0 . 0 .

(b) Further, $T_{0} \mathrm{FL}(s) \oplus \operatorname{Ker}(\pi \circ s)_{*}=T_{0} \mathbf{R}^{m}$ if and only if $Q\left\langle\tau_{F}^{*}\left(\omega_{h}\right), \tau_{F}^{*}\left(\omega_{h}\right)\right\rangle_{x} \neq$

Proof. We are given that $j_{0}^{1}(\pi \circ s)$ lies in ${ }_{1} S$. Notice that if $(\pi \circ s)_{*}$ has rank $m-1$ at 0 then $\operatorname{Ker}(\pi \circ s)_{*}$ is spanned by $s^{*} X_{h}$ and $\operatorname{coker}(\pi \circ s)_{*}=T_{x} M / \operatorname{Anhil}\left(\tau_{F}^{*}\left(\omega_{h}\right)\right)$. Recall that $j^{1}(\pi \circ s)$ induces, via $\left(j^{1}(\pi \circ s)\right)_{*}$, the intrinsic derivative, $D^{2}(\pi \circ s)$ : $T_{0} \mathbf{R}^{m} \rightarrow \operatorname{Hom}(\text { Ker, coker })_{0}$. The first assertion follows if $D^{2}(\pi \circ s)$ is onto, and the second follows if $D^{2}(\pi \circ S)\left(\operatorname{ker}(\pi \circ S)_{*}\right)_{0}$ is not the zero element of Hom(ker, coker). Let $\gamma:(\varepsilon,-\varepsilon) \rightarrow\left(\mathbf{R}^{m}, 0\right)$ be the trajectory of $s^{*} X_{h}$ passing through 0 . We need only show that $\left(d^{2} / d t^{2}\right)(\pi \circ s \circ \gamma)_{0} \neq 0$ for the first assertion, and that $\left\langle\tau_{F}^{*}\left(\omega_{h}\right)\right|\left(d^{2} / d^{2} t\right)(\pi \circ$ $\left.s \circ \gamma)_{0}\right\rangle_{x} \neq 0$ for the second. This will follow with a local calculation. Let $\left(x_{i}, p_{i}\right)$ be cotangent coordinates at $x$. Then the trajectories of $X_{h}$ satisfy $\dot{x}_{i}=h_{p_{i}}$, $\dot{p}_{i}=p_{i}-h_{x_{i}}$ for $i=1, \ldots, m$. Now $h_{p_{i}}\left(\tau_{F}(x)\right)=0$ for $i=1, \ldots, m$ and $\left(p_{i}-h_{x_{i}}\right)\left(\tau_{F}(x)\right) \neq 0$ for some $i$, otherwise $\tau_{F}(x)$ would be a point of contact singularity (both expressions denote a function evaluated at $\tau_{F}(x) \in T^{*} M$ ). Thus $\left(d^{2} / d^{2} t\right)(\pi \circ s \circ \gamma)_{0}$ is given by

$$
\begin{aligned}
\ddot{x}_{i}(0) \partial x_{i} & =\dot{p}_{j} h_{p_{i} p_{j}}\left(\tau_{F}(x)\right) \frac{\partial}{\partial x_{i}} \\
& =\left(p_{j}-h_{x_{j}}\right) h_{p_{i} p_{j}} \frac{\partial}{\partial x_{i}}={ }_{Q}\left\langle\tau_{F}^{*} \omega_{h},-\right\rangle_{x} .
\end{aligned}
$$

This does not vanish since $Q\langle\rangle$ is of full rank. For the second assertion we recall that $\tau_{F}^{*}\left(\omega_{h}\right)_{x}$ identifies with $\left(h_{x_{i}}\left(x, a_{j}(x)\right)-a_{i}(x)\right) d x_{i}$. Hence

$$
\begin{aligned}
\left\langle\tau_{F}^{*}\left(\omega_{h}\right) \mid \frac{d^{2}}{d^{2} t}(\pi \circ s \circ \gamma)_{0}\right\rangle_{x} & =\left\langle\left(h_{x_{k}}-p_{k}\right) d x_{k} \mid\left(p_{j}-h_{x_{j}}\right) h_{p_{i} p_{j}} \frac{\partial}{\partial x_{i}}\right\rangle \\
& =\bar{Q}\left\langle\tau_{F}^{*}\left(\omega_{h}\right), \tau_{F}^{*}\left(\omega_{h}\right)\right\rangle_{x}
\end{aligned}
$$

and we are finished.

Notice that (a) tells us that the PDE supplies transversality and that the condition in part (b) is on the PDE (compare examples 2 and 3). Also in the process of the above proof we have shown the following.

COROLlaRY 2.7. If $\tau_{F}(x) \in T^{*} M$ satisfies condition (b) above and $(\pi \circ s)_{*}$ has rank $m-1$ then $(\pi \circ s)$ is simply folded at 0 . Locally the image of $\pi \circ s$ lies to the side of Anhil $\left(\tau_{F}^{*}\left(\omega_{h}\right)\right)_{x}$ determined by $\bar{Q}_{Q}\left\langle\tau_{F}^{*}\left(\omega_{h}\right),-\right\rangle_{x} \in T_{x} M$.

CASE 4. Let $0 \neq \tau_{F}(x) \in T^{*} M$ be such that ${ }_{Q}\left\langle\tau_{F}, \tau_{F}^{*}\left(\omega_{h}\right)\right\rangle_{x} \neq 0, d_{Q} k(x) \neq 0$, and ${ }_{Q}\left\langle\tau_{F}^{*}\left(\omega_{h}\right), \tau_{F}^{*}\left(\omega_{h}\right)\right\rangle_{x} \neq 0$. It follows that $X_{h}$ is tangent to the fiber and that Anhil $\left(\omega_{h}\right)$ contains the tangent space to the fiber at $\omega_{h}(x)$.

THEOREM 2.8. Let $s:\left(\mathbf{R}^{m}, 0\right) \rightarrow\left(T^{*} M, \tau_{F}(x)\right)$ be the germ of a solution with $\tau_{F}(x)$ of Case 4. 
(a) The following is an open dense condition on $j_{0}^{2} s$.

(i) $(\pi \circ s)_{*}$ has rank $m-1$,

(ii) $s \pitchfork \tau_{F}$,

(iii) $d s^{*} h \neq 0$ when restricted to $T_{0} \mathrm{FL}(s)$,

(iv) $s_{*}(\mathrm{FL}(s)) \pitchfork V[h]$.

(b) For $s$ as in (a) the solution admits local models

$$
\begin{gathered}
(\pi \circ s)^{*}\left(x_{1}, \ldots, x_{m}\right)=\left(y_{1}, \ldots, y_{m-1}, y_{m}^{2}\right), \\
s^{*} h=y_{1}+y_{m}\left(\sum_{1}^{m} y_{i} q_{i}(y)\right), \quad q_{m}(0) \neq 0 .
\end{gathered}
$$

(c) For $s$ as in (a) $\operatorname{graph}(s):\left(\mathbf{R}^{m}, 0\right) \rightarrow\left(M \times \mathbf{R}, x \times h\left(\tau_{F}(x)\right)\right)$ fails to be immersive on a codimension 2 manifold of $\mathbf{R}^{m}$ contained in the fold locus of $s$. On this locus the rank of the graph germ is $m-1$.

ProOF. (a) Choose coordinates on $T^{*} M,\left(w, z, y_{i}, q_{i}\right), i=1, \ldots, m-1$, so that $\partial / \partial w$ represents $X_{h}, w=0$ represents $V[h]$, and $\lambda\left(d z-q_{i} d y_{i}\right)$ represents $\omega_{h}$. Notice that the cubic polynomials $P$ in $y_{1}, \ldots, y_{m-1}$ (with $d P=0$ at the origin) parametrize an open dense set of 2-jets of solutions $j_{0}^{1} s$. That is, if $P$ is such a polynomial then the associated 2 -jet is that of $s_{P}$ given by

$$
s_{P}^{*} w=w, \quad s_{P}^{*} z=P, \quad s_{P}^{*} y_{i}=y_{i}, \quad s_{P}^{*} q_{i}=\frac{\partial P}{\partial y_{i}} .
$$

Now the projection $\pi$ may be presented as $m$-functions $g_{1}, \ldots, g_{m}: \mathbf{R}^{2 m} \rightarrow \mathbf{R}$ with $\partial g_{i} / \partial w=0$, and $d g_{1} \wedge \cdots \wedge d g_{m} \neq 0$ at the origin. Now $T_{0} \mathrm{FL}(s)$ is given by $\operatorname{Anhil}\left(d_{0} f\right)$ where

$$
s_{P}^{*}\left(d g_{1} \wedge \cdots \wedge d g_{m}\right)=f d w \wedge d y_{1} \wedge \cdots \wedge d y_{m-1},
$$

and the condition that $s_{P}$ fail to satisfy (i), (ii), (iii), (iv) may be written as the vanishing of a list of polynomials in the coefficients of $P$. One readily sees that these polynomials are not trivial.

(b) The construction of the local model for $(\pi \circ s)$ may be found in Golubitsky and Guillemin. Since $s^{*} h$ restricts to the fold locus of $(\pi \circ s)$ with nonvanishing differential we may modify the coordinates given in the fold model so that $s^{*} h$ is represented by $y_{1}+y_{m} q$. Since $s^{*} X_{h}(0) \sim \partial / \partial y_{m}$ and $\tau_{F} \subset V[h]$ we see that $q$ and $\partial q / \partial y_{m}=0$ at 0 , and $\partial^{2} q / \partial y_{m} \partial y_{m} \neq 0$ at 0 .

(c) Using the model constructed above we may identify the locus where the graph fails to be immersive with the variety $\left\{y_{m}=0, \partial s^{*} h / \partial y_{m}=0\right\}$. But $\left\langle X_{h}^{2} \mid h\right\rangle \neq 0$ implies $\partial^{2} /\left.\partial y_{m} \partial y_{m} s^{*} h\right|_{0} \neq 0$ (recall Proposition 2.5 and that $s^{*} X_{h} \sim \partial / \partial y_{m}$ at $0)$. Part (c) is now an immediate consequence.

RETURN TO CASE 3. Let $o_{x}=\tau_{F}(x) \in T^{*} M$ be such that $d_{A} k(x) \neq 0$, ${ }_{A} Y(x)=0$, and End ${ }_{A} Y(X)_{x}=\left[X,{ }_{A} Y\right]_{x}$ is an automorphism of $T_{x} M$. Further assume that $Q\left\langle\tau_{F}^{*}\left(\omega_{h}\right), \tau_{F}^{*}\left(\omega_{h}\right)\right\rangle_{x} \neq 0$ and ${ }_{Q}\left\langle d_{Q} k, d_{Q} k\right\rangle_{x} \neq 0$. A computation shows that Anhil $\left(\omega_{h}\right)$ and $X_{h}^{\perp}(\perp$ relative to $\odot$ Hess $\theta)$ agree as subspaces of $T_{o_{x}} T^{*} M$. Thus $s^{*} X_{h}$ lies in the radical of Hess $s_{o} s^{*} h$ for all solution germs $s:\left(\mathbf{R}^{m}, 0\right) \rightarrow$ $\left(T^{*} M, o_{x}\right)$. Futher $V[h]$ is a singular variety at $o_{x}$. The automorphism condition on End ${ }_{A} Y$ implies that $\left\langle X_{h} \mid h\right\rangle$ is morse at $o_{x}$. Since $Z \subset V[h]$ we conclude that $V[h]$ has the structure of a type $(m, m)$ quadratic cone. It is readily shown that ${ }_{A} k(x)={ }_{Q} k(x), d_{A} k(x)=d_{Z} k(x)$, and End ${ }_{A} Y=\operatorname{End}_{Q} Y$, at $x$. 
THEOREM 2.9. Let $s:\left(\mathbf{R}^{m}, 0\right) \rightarrow\left(T^{*} M, o_{x}\right)$ be the germ of a solution with $o_{x}$ of Case 3.

(a) The following is an open dense condition on $j_{0}^{2} s$.

(i) $s$ is transverse to $Z$ and $\tau_{F}$,

(ii) $(\pi \circ s)_{*}$ is of rank $m-1$,

(iii) Hess $s^{*} h$ is of rank $m-1$,

(iv) $s^{*} \operatorname{Hess}\left\langle X_{h} \mid h\right\rangle$ is of rank $m$,

(v) $s^{*} \operatorname{Hess}\left\langle X_{h} \mid h\right\rangle$ restricted to $T_{0} \mathrm{FL}(s)$ is of rank $m-1$.

(b) For $s$ as in (a), the solution admits local model

$$
\begin{aligned}
& (\pi \circ s)^{*}\left(x_{1}, \ldots, x_{m}\right)=\left(y_{1}, \ldots, y_{m-1}, y_{m}^{2}\right), \\
& s^{*} h=\sum_{1}^{m-1} \pm y_{i}^{2}+y_{m}\left(\sum_{1}^{m} y_{i} y_{j} q_{i j}(y)\right),
\end{aligned}
$$

with $q_{m m}(0) \neq 0$.

(c) For $s$ as in (a) the graph of $s$ fails to be an immersion on codimension 2 variety which lies in the fold locus of $s$. This variety has the structure of a quadratic cone. Its quadratic type is that of $s^{*} \operatorname{Hess}\left\langle X_{h} \mid h\right\rangle$ restricted to $T_{0} \mathrm{FL}(s)$.

(d) Let $s_{u}$ be a deformation such that $s_{0}$ is as in (a). If $j_{(0,0)}^{1} s_{u}$ satisfies an open dense condition: $\left(X_{h} \oplus s_{u *}(\partial / \partial u)\right.$ spans a type $(1,1) 2$-plane relative to Hess $\left.\left\langle x_{h} \mid h\right\rangle\right)$, then for $u \neq 0$,

(i) $\pi \circ s_{u}$ is folded simply,

(ii) $s_{u}^{*} h$ has two morse critical points with types $(r+1, s)$ and $(r, s+1)$. One critical point is contained in $\mathrm{FL}\left(s_{u}\right)$, the other is at a submersion point of $\pi \circ s_{u}$.

PROOF. (a) Left to the reader; compare the proof of Case 3(a).

Parts (b) and (c) follow by the arguments found in parts (b) and (c) of Case 3.

(d) Since $s_{u}$ as a map of $\mathbf{R}^{m} \times \mathbf{R}$ is transverse to $Z$ and $\tau_{F}$ the preimages of these submanifolds will be curves $c(Z)$ and $c\left(\tau_{F}\right)$ in $\mathbf{R}^{m} \times \mathbf{R}$. We may assume $s_{u}$ is parametrized so that $s_{u}(0)$ describes $c(Z)$. Now we choose coordinates so that $s_{0}$ is modeled by Case $3(\mathrm{~b})$. Consider the submanifold $\left\{\left(\partial / \partial y_{i}\right)\left(s_{u}^{*} h\right)=0\right.$, $i=1, \ldots, m-1\}$ in $\mathbf{R}^{m} \times \mathbf{R}$. We claim that $s^{*} X_{h}$ and $\partial / \partial u$ span its tangent space at $(0,0)$. To see this, observe that:

$$
\left.s^{*} X_{h}\left(\frac{\partial}{\partial y_{i}}\left(s_{u}^{*} h\right)\right)\right|_{0,0}=\left.X_{h}\left(\frac{\partial}{\partial y_{i}}\left(s_{0}^{*}(h)\right)\right)\right|_{0}=\left.\frac{\partial}{\partial y_{i}}\left(s_{0}^{*}\left\langle X_{h} \mid h\right\rangle\right)\right|_{0}=0,
$$

since $d\left\langle X_{h} \mid h\right\rangle=0$ at $o_{x}$. Further, $\left(\partial / \partial y_{i}\right)\left(s_{u}^{*} h\right)=0$ when restricted to $(0, u)$ since $s_{u}(0) \simeq c(Z)$. Now recall that Hess $\left\langle X_{h} \mid h\right\rangle$ is of maximal rank. Since $Z \subset V[h]$ we see that the condition: $X_{h} \oplus\left(s_{u}\right)_{*}(\partial / \partial u)$ is a type $(1,1) 2$-plane in $T_{o_{x}} T^{*} M$ (relative to Hess $\left.\left\langle X_{h} \mid h\right\rangle\right)$, is an open dense condition on the 1-jet of the deformation. With this assumption we see that $\left\{\left(\partial / \partial y_{i}\right) s_{u}^{*} h=0, i=1, \ldots, m\right\}$ identifies with two curves $c(Z)$ and $c$ in $\mathbf{R}^{m} \times \mathbf{R}$. (In general the curves $c(Z), c\left(\tau_{F}\right), c$ will be distinct. See example 2.) Now $X_{h}$ is not tangent to $Z$ at $\tau_{F}(x)$ so $c$ cannot be tangent to $Z$. Since $s_{u}^{*} h=s_{u}^{*} \theta$ it follows that the critical point of $s_{u}^{*} h$ corresponding to $c$ must occur at a point where $\pi \circ s_{u}$ is singular. Observe that the tangent space to $j^{1}\left(\pi \circ s_{u}\right)^{-1}\left({ }_{1} S\right)$ at the origin of $\mathbf{R}^{m} \times \mathbf{R}$ is given by $T c \oplus T \mathrm{FL}\left(s_{0}\right)$. Since $c(Z)$ is transverse to this subspace we see that for fixed $u$ the critical point of $s^{*} h$ corresponding to $c(Z)$ is a submersion point of $\pi \circ s_{u}$. 


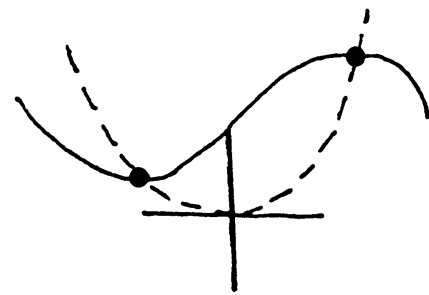

$u>0$

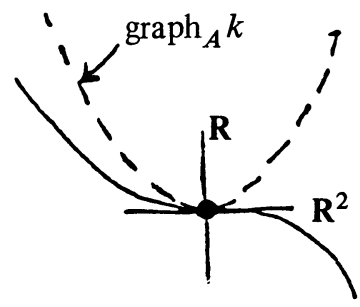

$u=0$

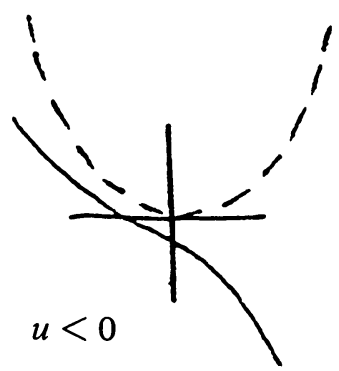

DIAGRAM 1.1

Turning to the signature of these critical points we need only discuss the behavior of $s_{u}^{*}(h)$ restricted to the 2-dimensional submanifold $\left\{\left(\partial / \partial y_{i}\right) s_{u}^{*} h=0 \mid i=\right.$ $1, \ldots, m-1\}$. Let $i:\left(\mathbf{R}^{2}, 0\right) \rightarrow\left(\mathbf{R}^{m} \times \mathbf{R}, 0\right)$ be such that $i(0, u)$ parametrizes $c(Z)$ and $i(v, 0)$ parametrizes $c$. Now we compute

$$
\left\langle\frac{\partial}{\partial u} \mid i^{*}\left(\frac{\partial^{2}}{\partial y \partial y} s_{u}^{*} h\right)\right\rangle \text { and }\left\langle\frac{\partial}{\partial v} \mid i^{*}\left(\frac{\partial^{2}}{\partial y \partial y} s_{u}^{*} h\right)\right\rangle,
$$

at the origin of $\mathbf{R}^{2}$. But this identifies with $i^{*} \operatorname{Hess}\left\langle X_{h} \mid h\right\rangle$ paired with $\left(\partial / \partial u \odot s^{*} X_{h}\right)$ and $\left(\partial / \partial v \odot s^{*} X_{h}\right)$ respectively. (Recall that $s^{*} X_{h}$ lies in the image of $\left.\left(i_{*}\right)_{0}.\right)$ Now relative to $\operatorname{Hess}\left\langle X_{h} \mid h\right\rangle, T c(Z)$ and $T c$ at $o_{x}$ are the null directions of a type $(1,1)$ plane. We conclude that both of these expressions are nonzero. Thus the signature changes along each curve. By transversality the types must change from $(r+1, s)$ to $(r, s+1)$.

3. Examples. Two examples illustrating Cases 1-4 will be given. A third example illustrates that Cases 1-4 are not exhaustive. In all of these $M$ will be the plane $\mathbf{R}^{2}$.

1. Consider the PDE $f=x^{2} \pm y^{2}+f_{x}$ so that $h=x^{2} \pm y^{2}+p$. We see that

$$
\omega_{h}=(2 x-p) d x+(2 y-q) d y+d p,
$$

so that ${ }_{A} k=x^{2}+y^{2},{ }_{A} Y=\partial x$. It follows that $o_{0} \simeq(0,0,0,0) \in T^{*} \mathbf{R}^{2}$ is an isolated Case 2 point. The remaining points on $Z \simeq(x, y, 0,0)$ are of Case 1 . We see that there is no folding in the PDE. As a deformation of solutions we may take $s_{u}=d f_{u}$ with

$$
f_{u}(x, y)=x^{2}+2 x+2+e^{x} a(y, u),
$$

where $a(0,0)=-2$ and $(\partial / \partial y) a(0,0)=0$. The assumption in Case 2(a) reads as $\left(\partial^{2} / \partial^{2} y\right) a(0,0) \neq 0$. Since $V[h]$ corresponds to $p=0$ in $T^{*} M$ we see that $\left(S_{u}\right)_{*}(\partial / \partial u) \pitchfork V[h]$ if $(\partial a / \partial u)(0,0) \neq 0$.

We visualize the bifurcation in the case $a=u+y^{2}-2$. The graph of ${ }_{A} k$ in $M \times \mathbf{R}$ is the image of $E \cap C J$ under $\pi_{M \times \mathbf{R}}$. Thus all the critical points of $f_{u}$ will lie on the graph of ${ }_{A} k$. Suppressing one dimension we may visualize matters in $M \times \mathbf{R}$ as in Diagram 1 .

2. Consider the PDE

$$
f=x+y+x f_{x}+y f_{y}+f_{x / 2}^{2}+f_{y / 2}^{2}
$$

so that in cotangent coordinates $(x, y, p, q)$ on $T^{*} \mathbf{R}^{2}$,

$$
h=x+y+x p+y q+p^{2} / 2+q^{2} / 2 \text {. }
$$


We see that

$$
\begin{aligned}
& \omega_{h}=d x+d y+(p+x) d p+(q+y) d q, \\
& \tau_{F}=-\frac{1}{2} d\left(x^{2}+y^{2}\right), \\
& \tau_{F}^{*}(h)={ }_{Q} k=x+y-\frac{1}{2}\left(x^{2}+y^{2}\right), \\
& { }_{Q}\langle\rangle=\frac{1}{2}\left(d x^{2}+d y^{2}\right), \\
& { }_{Q} Y=\left(x \frac{\partial}{\partial x}+y \frac{\partial}{\partial y}\right) .
\end{aligned}
$$

Thus the PDE is folded simply, and $o_{0}$ is the only point of Case 3. Points on $\tau_{F}$ sufficiently near $\tau_{F}(0,0)$ are of Case 4.

We now compute

$$
\begin{aligned}
& \tau_{F}^{*}\left(\omega_{h}\right)=d(x+y), \quad{ }_{Q}\left\langle\tau_{F}^{*}\left(\omega_{h}\right), \tau_{F}^{*}\left(\omega_{h}\right)\right\rangle=1, \\
& -Q\left\langle\tau_{F}^{*}\left(\omega_{h}\right),-\right\rangle=-\frac{1}{2}\left(\frac{\partial}{\partial x}+\frac{\partial}{\partial y}\right) .
\end{aligned}
$$

It follows that any solution intersecting $\tau_{F}$ with $(\pi \circ s)_{*}$ of rank 1 is folded simply with image of $\pi \circ s$ lying on the $-\partial / \partial x-\partial / \partial y$ side of Anhil $\left(\tau_{F}^{*} \omega_{h}\right)$.

Let us discuss an explicit deformation of solutions for this PDE. Consider $s_{u}: \mathbf{R}^{2}$ $\times \mathbf{R} \rightarrow T^{*} \mathbf{R}^{2}$ given in cotangent coordinates $(x, y, p, q)$ by

$$
s_{u}(a, b)=\left(1-a-f_{u} e^{-a}, 1+\operatorname{Ln} f_{u}^{\prime}-a+b f_{u}^{\prime} e^{-a}, a, a-\operatorname{Ln} f_{u}^{\prime}\right),
$$

where $f_{u}=f(b)+u$. Here $f(b)$ is any smooth real valued function with $f(-1)=$ $f^{\prime}(-1)=1$. (In the text we have written $s_{u}^{*} x=1-a-f_{u} e^{-q}, s_{u}^{*} p=a, \ldots$, etc. for $s_{u}(a, b)$.) Now for each fixed $u, s_{u}$ is a solution and $s_{0}(0,-1)=(0,0,0,0)=o_{0} \in$ $T^{*} \mathbf{R}^{2}$ so that $s_{0}(0,-1)$ is a point of Case 3 . Also, up to a $C^{\infty}$ multiple $s_{u}^{*} X_{h}$ is given by $\partial / \partial a$.

Let us see when $s_{u}$ satisfies the conditions listed in (a) of Theorem 2.9.

(i) Since $T Z=T \tau_{F}$ at $o_{0}$ we compute the representatives of $\left(s_{0}\right)_{*}$ to be

$$
\left(\begin{array}{cc}
* & * \\
* & * \\
1 & 0 \\
1 & -f^{\prime \prime} / f^{\prime}
\end{array}\right)
$$

and conclude that (i) is satisfied iff $f^{\prime \prime}(-1) \neq 0$. (We will see that the remaining conditions (ii) $-(\mathrm{v})$ also follow from $f^{\prime \prime}(-1) \neq 0$.)

(ii) We compute the representative of $\left(\pi \circ s_{0}\right)_{*}$ to be

$$
\left(\begin{array}{ll}
-1+f e^{-a} & -f^{\prime} e^{-a} \\
-1-b f e^{-a} & f^{\prime \prime} / f^{\prime}+f^{\prime} e^{-a}+b f^{\prime \prime} e^{-a}
\end{array}\right) .
$$

Evaluating at $(0,-1)$ we get $\left(\begin{array}{l}0 * \\ 0 *\end{array}\right)$ which is of rank one with $s_{0}^{*} X_{h} \sim \partial / \partial a$ spanning its kernel. Also, one computes $d$ (Det) evaluated at $(0,-1)$ to be $-2 d a+f^{\prime \prime}(-1) d b$. Hence $T_{(0,-1)} \mathrm{FL}\left(s_{0}\right)$ is spanned by $f^{\prime \prime}(-1)(\partial / \partial a)+2(\partial / \partial b)$. This directly verifies that $\pi \circ s_{0}$ is simply folded at $(0,-1)$ iff $(\pi \circ s)_{*}$ has rank 1 at 0 (compare Corollary $2.7)$. 

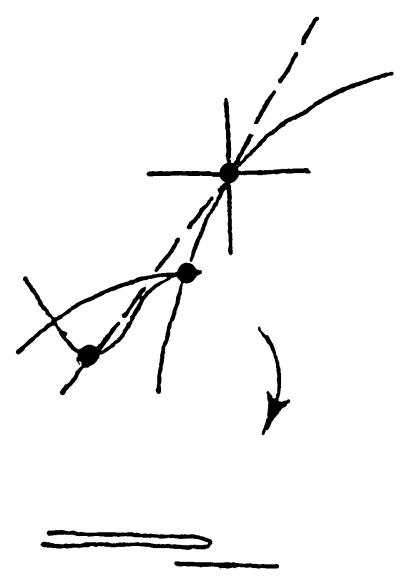

$u<0$

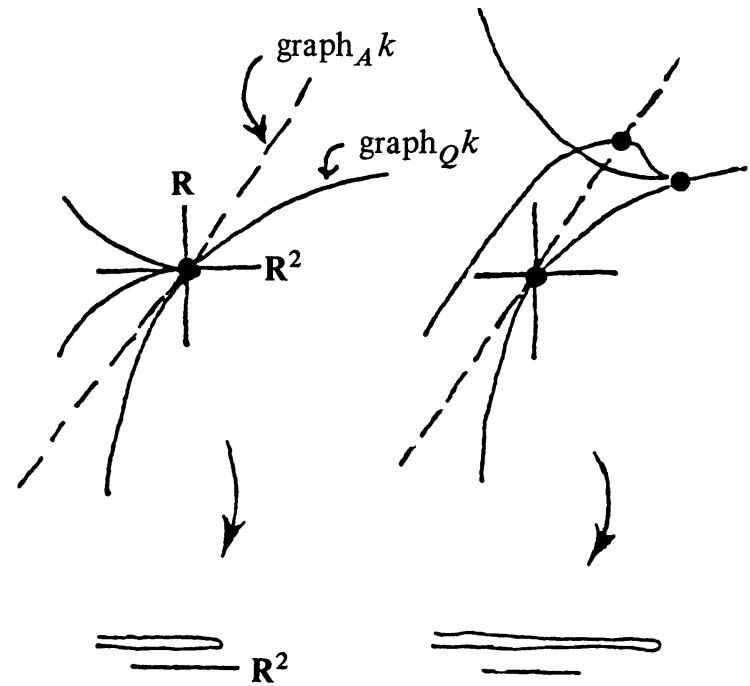

$u=0$ $u>0$

\section{DIAGRAM 2.1}

(iii) It is immediate that Hess $s_{0}^{*} h$ has at most rank 1. We compute that

$$
s_{0}^{*} h(0, b) \equiv \frac{f^{\prime \prime}}{f^{\prime}}(b+1) \bmod \mathcal{M}^{2}(\mathbf{R}) .
$$

If $f^{\prime \prime}(-1) \neq 0$, it follows that $\left.\left(\partial^{2} / \partial b^{2}\right) s_{0}^{*} h\right|_{(0,1)} \neq 0$ and hence that Hess $s_{0}^{*} h$ has rank 1.

(iv) and (v). We compute that $s_{0}^{*} \operatorname{Hess}\left\langle X_{h} \mid h\right\rangle$ evaluated at $(0,-1)$ is represented by $\left(\begin{array}{ll}0 & 1 \\ 1 & 0\end{array}\right)$. Since $T_{(0,1)} \mathrm{FL}\left(s_{0}\right)$ is spanned by $f^{\prime \prime}(-1)(\partial / \partial a)+2(\partial / \partial b)$ it follows that Hess $s^{*}\left\langle X_{h} \mid h\right\rangle$ restricted to $T_{(0,1)} \mathrm{FL}\left(s_{0}\right)$ has maximal rank. This directly verifies that $\operatorname{graph}\left(s_{0}\right)$ fails to be immersive at the isolated point $(0,-1)$ (compare Theorem 2.9 , part (c)).

Let us examine the deformation. It is clear that $s_{u}(0,-1)$ parametrizes $c(Z)$ (the curve of critical points of $s_{u}^{*} h$ which are also submersion points of $\left.\pi \circ s_{u}\right)$. Now we compute that Hess $s^{*}\left\langle X_{h} \mid h\right\rangle\left(X_{h}, X_{h}\right)=0$ and Hess $s^{*}\left\langle X_{h} \mid h\right\rangle\left(X_{h}, s_{u *}(\partial / \partial u)\right)=-1$, at $(0,-1)$. Hence $X_{h} \oplus s_{u *}(\partial / \partial u)$ spans a type $(1,1) 2$-plane relative to $\operatorname{Hess}\left\langle X_{h} \mid h\right\rangle$. Since $\tau_{F} \pitchfork Z$ it is clear that the curves $c(Z), c\left(\tau_{F}\right)$, and $c$ are distinct. To visualize the bifurcation observe that the graphs of ${ }_{A} k$ and ${ }_{Q} k$ in $\mathbf{R}^{2} \times \mathbf{R}$ are the images of $Z$ and $\tau_{F}$ under $\pi_{M \times \mathbf{R}} \circ G(h)$. Thus the images of the curves $c(Z)$ and $c\left(\tau_{F}\right)$ will be contained in these graphs. Now $\pi \circ s_{u}\left(\mathrm{FL}\left(s_{u}\right)\right)$ will divide $\mathbf{R}^{2}$ into two components. The graph of $s_{u}$ will double cover the component on the $-\partial / \partial x-\partial / \partial y$ side of $\left(\pi \circ s_{u}\right)\left(\mathrm{FL}\left(s_{u}\right)\right)$. Suppressing one dimension we may visualize matters in $\mathbf{R}^{2} \times \mathbf{R}$ as in Diagram 2.

Let us examine some solutions of this PDE which do not satisfy conditions (i)(v). Consider the solution $s: \mathbf{R}^{2} \rightarrow T^{*} \mathbf{R}^{2}$ given by

$$
s(a, b)=\left(1-a-e^{-a}, 1-b-e^{-b}, a, b\right) .
$$


Here $s(0,0)=o_{0}=\tau_{F}(0,0)$ and $(\pi \circ s)_{*}$ has rank zero at $(0,0)$. Notice that $\pi \circ s$ has a fold at every point in the set $\{(a, 0)\} \cup\{(0, b)\}-\{(0,0)\}$ and that the image of $s$ on this set is disjoint from $\tau_{F}$. The graph of $s$ fails to be immersive only at $(0,0)$. Consider the solutions

$$
s_{u}(a, b)=\left(1-a+b-e^{-a}, 1+u-a-b-e^{-a}, a-b, a+b-u\right) .
$$

Here each $s_{u}$ intersects $\tau_{F}$ along a curve which $\pi$-projects onto $x+y=u$. Notice that $\pi \circ s_{u}$ is folded along this curve and the image of $\pi \circ s_{u}$ double covers the set $\left\{(x, y) \in \mathbf{R}^{2} \mid x+y<u\right\}$. The graph of $s$ fails to be immersive exactly on the points which correspond to the curve $s_{u} \cap \tau_{F}$.

We close our discussion of this PDE with a simple observation.

PROPOSITION 3.1. There exists no (global) solution $f: \mathbf{R}^{2} \rightarrow \mathbf{R}$ to the PDE in Example 2 which satisfies the following:

(i) $f$ has a critical point at $(x, y)$,

(ii) $\operatorname{Hess}_{(x, y)} f$ is umbilic, and positive definite (relative to ${ }_{Q}\langle\rangle_{x, y}$ ).

(Note: there exist local solutions which satisfy these conditions.)

PROOF. If such a solution existed then the trajectory of $X_{h}$ which contains $d_{(x, y)} f \in T^{*} \mathbf{R}^{2}$ also intersects $\tau_{F}$. But then the solution cannot have $(\pi \circ s)_{*}$ at full rank at this point, contradiction.

3. Consider the PDE

$$
f=x+y+x f_{x}+y f_{y}+f_{x / 2}^{2}-f_{y / 2}^{2} .
$$

We see that

$$
\tau_{F}=-x d x+y d y, \quad \tau_{F}^{*} \omega_{h}=d x+d y, \quad Q\langle\rangle=d x^{2}-d y^{2} ;
$$

thus the PDE is folded simply. But ${ }_{Q}\left\langle\tau_{F}^{*} \omega_{h}, \tau_{F}^{*} \omega_{h}\right\rangle=0$ on all of $\mathbf{R}^{2}$. It follows by the arguments of Theorem 2.6 that no solution $s: L \rightarrow T^{*} M$ which intersects $\tau_{F}$ has a fold singularity in $\pi \circ s$ (at the point corresponding to $s \cap \tau_{F}$ ). One can also show that any solution which intersects $\tau_{F}$ at a point over the set $x+y=0$ in $\mathbf{R}^{2}$ cannot have $\pi \circ s$ transverse to ${ }_{1} S$ in $J^{1}\left(\mathbf{R}^{2}, \mathbf{R}^{2}\right)$. Thus the geometry of the PDE forces instability of all solutions which contain such a point of $\tau_{F}$.

\section{REFERENCES}

[A] V. I. Arnold, Geometrical methods in the theory of ordinary differential equations, Springer-Verlag, 1983.

[B] R. Bryant, S. S. Chern, and P. Griffiths, Exterior differential systems, preprint.

[G] R. B. Gardner, Lectures on exterior differential systems, UNC, 1980.

[GG] M. Golubitsky and V. Guillemin, Stable mappings and their singularities, Graduate Texts in Math., vol. 14, Springer-Verlag, Berlin and New York, 1973.

[GJ] J. Guckenhiemer, Catastrophes and PDE, Ann. Inst Fourier (Grenoble) 23 (1973).

[GS] V. Guillemin and S. Sternberg, Geometric asymptotics, Math. Surveys, no. 14, Amer. Math. Soc., Providence, R. I., 1977.

[K] M. Kossowski, First order PDE with singular solutions, Indiana Univ. Math. J. (to appear).

[L] V. V. Lychagin, Local classification of non-linear first order P.D.E., Russian Math. Surveys 30 (1975), 105-175.

\section{Department of Mathematics, Rice University, Houston, Texas 77001}

Current address: Department of Mathematics, University of South Carolina, Columbia, South Carolina 29208 